\title{
Variability of immunohistochemical reactivity on stored paraffin slides
}

Philippe Bertheau, Dominique Cazals-Hatem, Véronique Meignin, Anne de Roquancourt, Olivier Vérola, Agnès Lesourd, Carmen Séné, Claude Brocheriou, Anne Janin

\begin{abstract}
Aims-To investigate the effects of slide storage on immunohistochemical staining, since recent reports have indicated that storage of unstained paraffin slides for up to 12 weeks may lead to false negative immunostaining of tumour markers. Methods-11 antibodies (anti-cytokeratin, epithelial membrane antigen (EMA), vimentin, smooth muscle actin, PS100, chromogranin, CD45, CD20, CD3, CD30, and oestrogen receptor $(\mathrm{OR})$ ) were tested on unstained paraffin slides of breast carcinomas, lymphomas, and neuroendocrine tumours that had been stored for three to 10 years. All the paraffin blocks were recut less than one week before immunostaining. Immunostainings of years old slides were compared with those of recent slides in at least five cases for each antibody. For three antibodies (antichromogranin, anti-CD3, and anti-OR) we also tested one year old and three months old slides.

Results-Intensity of staining on years old slides was strikingly reduced for chromogranin and CD3 in several cases and was slightly stronger for vimentin. In some cases a significant decrease of $O R$ positivity was observed after three months storage, and a complete loss of OR immunostaining after 12 months. No significant difference was noted with the other antibodies.
\end{abstract}

Conclusions-Immunohistochemical detection of some antigens located either in the nucleus, in the cytoplasm, or on the cytoplasmic membrane could be impaired by storage of paraffin slides as short a time as three months. One should be cau-

Pathology, Hospital Saint-Louis, Paris,

France

P Bertheau

D Cazals-Hatem

V Meignin

A de Roquancourt

$O$ Vérola

A Lesourd

C Séné

C Brocheriou

A Janin

Correspondence to: Dr Philippe Bertheau, Service d'Anatomie Pathologique, Hôpital Saint-Louis, 1 avenue Claude Vellefaux, 75010 Paris, France; email jophbert@micronet.fr

Table 1 Characteristics of the antibodies

\begin{tabular}{llll}
\hline Antibody & Origin & Type & Dilution \\
\hline KL1 & Immunotech $†$ & $\mathrm{M}$ & $1: 200$ \\
EMA & Dako $\ddagger$ & $\mathrm{M}$ & $1: 50$ \\
Vimentin & Dako & $\mathrm{M}$ & $1: 50$ \\
SMA & Sigma & $\mathrm{M}$ & $1: 2000$ \\
PS100 & Dako & $\mathrm{P}$ & $1: 1500$ \\
Chromogranin & Dako & $\mathrm{M}$ & $1: 50$ \\
CD45 & Dako & $\mathrm{M}$ & $1: 100$ \\
CD20 & Dako & $\mathrm{M}$ & $1: 200$ \\
CD3 & Dako & $\mathrm{M}$ & $1: 50$ \\
CD30 & Dako & $\mathrm{M}$ & $1: 50$ \\
OR & Novocastra $\$$ & $\mathrm{M}$ & $1: 20$ \\
\hline
\end{tabular}

${ }^{\star} \mathrm{M}$, monoclonal; $\mathrm{P}$, polyclonal.

†Marseille, France.

ISt Louis, Missouri, USA

@Newcastle, UK.

EMA, epithelial membrane antigen; KL1, cytokeratin; OR, oestrogen receptor clone $6 \mathrm{~F} 11$; SMA, smooth muscle actin. tious of doing retrospective immunohistochemical studies on stored unstained slides.

(F Clin Pathol 1998;51:370-374)

Keywords: unstained slides; stored slides; immunohistochemistry; paraffin slides

Tissue preservation is a major concern for everyone undertaking morphological studies. Biological samples may be frozen and stored at very low temperature, allowing good preservation of tissues even for years. ${ }^{1}$ Immersion of specimen in fixative solutions is the most commonly used method of tissue preservation in pathology laboratories. Once fixed, the specimen is embedded in paraffin and can be stored for many years. Unstained slides are usually prepared from the paraffin blocks one or two days before immunostaining. However, blocks are sometimes no longer available and immunohistochemistry has to be performed on unstained slides that had been prepared some time before. Until recently no one had questioned the quality of the results obtained with such old unstained slides. It was nevertheless recommended that one should not use very old unstained slides, though what constituted "too old" was not clearly defined. Recent studies ${ }^{2}{ }^{3}$ have reported strong decrease of p53 immunoreactivity in breast cancer after a 12 weeks storage of the unstained slides.

We have tested immunohistochemistry with 11 antibodies on unstained slides that had been stored from three months to 10 years.

\section{Methods}

We retrieved from our pathological files cases of breast cancer, lymphoma, and neuroendocrine tumours diagnosed between 1986 and

Table 2 Immunostaining in years old slides compared with recent slides

$\ddagger$ Trappes, France.

\begin{tabular}{lllll}
\hline & \multirow{2}{*}{$\begin{array}{c}\text { Cases with modified } \\
\text { staining/cases tested }(n / n) *\end{array}$} & \multicolumn{3}{l}{ Results of comparisons $\dagger$} \\
\cline { 3 - 5 } & & Neg & $<$ & $<$ \\
\hline Chro & $4 / 5$ & 0 & 3 & 1 \\
CD3 & $5 / 9$ & 1 & 1 & 3 \\
PS100 & $1 / 5$ & 0 & 0 & 1 \\
CD30 & $1 / 6$ & 0 & 0 & 1 \\
KL1 & $0 / 5$ & 0 & 0 & 0 \\
EMA & $0 / 5$ & 0 & 0 & 0 \\
SMA & $0 / 5$ & 0 & 0 & 0 \\
CD45 & $0 / 5$ & 0 & 0 & 0 \\
CD20 & $0 / 5$ & 0 & 0 & 0 \\
Vim & $6 / 8$ & & &
\end{tabular}

${ }^{\star} \mathrm{n}$, number of cases; reduced staining for all antigens except vimentin, which was moderately increased.

$\nmid N e g$, no staining in older slide; $<<$, strongly reduced staining intensity; $<$, moderately reduced staining intensity.

Chro, chromogranin; EMA, epithelial membrane antigen; KL1, cytokeratin; SMA, smooth muscle actin; Vim, vimentin. 
Table 3 Oestrogen receptor (OR), chromogranin (Chro), and CD3 immunostaining in one year old slides and three month old slides compared with recent slides

\begin{tabular}{|c|c|c|c|c|}
\hline & \multirow{2}{*}{$\begin{array}{l}\text { Cases with modified staining/cases } \\
\text { tested }(n / n)^{\star}\end{array}$} & \multicolumn{3}{|c|}{ Results of comparisonst } \\
\hline & & $\mathrm{Neg}$ & $<<$ & $<$ \\
\hline OR, 1 year & $5 / 6$ & 4 & 1 & 0 \\
\hline OR, 3 months & $5 / 8$ & 1 & 4 & 0 \\
\hline Chro, 1 year & $3 / 6$ & 0 & 0 & 3 \\
\hline Chro, 3 months & $1 / 7$ & 0 & 0 & 1 \\
\hline $\mathrm{CD} 3,1$ year & $1 / 5$ & 0 & 0 & 1 \\
\hline CD 3,3 months & $0 / 5$ & 0 & 0 & 0 \\
\hline
\end{tabular}

$\star \mathrm{n}$, number of cases.

$\nmid \mathrm{Neg}$, no staining in older slide; $<<$, strongly reduced staining intensity; $<$, moderately reduced staining intensity.

1995. All tissues had been fixed in $10 \%$ formalin for 18 to 24 hours and postfixed in Bouin's solution for four to six hours until further processed for paraffin embedding. We selected cases for which unstained slides had been stored. We studied five to nine cases for each antibody tested.

\section{STUDY OF YEARS OLD SLIDES}

Five micrometre sections had been cut and mounted on standard slides without adhesive pretreatment. Unstained slides were stored with stained slides in metallic drawers under safe light conditions with no air conditioning system, room temperature fluctuating from $19^{\circ} \mathrm{C}$ to $25^{\circ} \mathrm{C}$. The same blocks were recut in 1996 with identical conditions of section thickness and types of slide. Immunohistochemical techniques were performed simultaneously on years old slides and recent slides within seven days after recutting. We compared immunostaining of years old slides and recent slides for all 11 antibodies except anti-oestrogen receptors (OR), which need gelatin coated slides.

STUDY OF ONE YEAR OLD AND THREE MONTHS OLD SLIDES

For some cases, we recut the blocks in 1996 and stored the unstained slides for one year. We prepared both gelatin coated slides and nongelatin-coated slides. We recut the blocks nine months later and stored the slides for three months. Then we had the blocks recut a final time and immunohistochemistry was performed simultaneously on one year old, three months old, and recent slides.

Among the 11 antibodies, we tested antiOR, which needed microwave pretreatment on gelatin coated slides, and anti-chromogranin and anti-CD3, which showed a strong decrease in immunoreactivity in slides stored for years.

SECTION PRETREATMENT

Tissue sections were deparaffinised and rehydrated. Endogenous peroxidase was blocked with $3 \% \mathrm{H}_{2} \mathrm{O}_{2}$ for 20 minutes.

For OR and CD3 immunostainings on one year old and three months old gelatin coated slides, we subjected the sections to antigen retrieval by heating the slides in a microwave oven in $10 \mathrm{mM}$ citrate buffer, $\mathrm{pH} 7$, for a total of 10 minutes (two five minute periods with replacement of evaporated water).

For CD3, CD20, CD30, vimentin, and EMA immunostaining on years old nonpretreated slides, we immersed the slides 10 minutes in $60^{\circ} \mathrm{C}$ preheated $10 \mathrm{mM}$ citrate buffer, $\mathrm{pH} 7$. Using this technique, only a few sections detached from the slides and antigen retrieval was acceptable.

Other antibodies (anti-cytokeratin, smooth muscle actin, PS100, CD45, chromogranin) did not require section pretreatment.

\section{IMMUNOHISTOCHEMISTRY}

Old and recent slides from each block were processed simultaneously with the same protocol. Polyclonal and monoclonal antibodies used in this study are listed in table 1.

Cytokeratin (KL1), epithelial membrane antigen (EMA), vimentin, smooth muscle actin (SMA), PS100, and oestrogen receptor (OR) immunostaining was tested on sections of breast carcinoma. CD 45, CD20, CD3, and CD30 immunostaining was tested on various types of lymphomas of the $\mathrm{B}$ or $\mathrm{T}$ cell phenotype. Immunostaining with chromogranin was tested on phaeochromocytomas.

All incubations were performed at room temperature as follows:

-Primary antibodies were incubated for one hour.

-Link antibody was incubated for $30 \mathrm{~min}$ utes.

-Peroxidase conjugated third antibody or mouse alkaline phosphatase anti-alkaline phosphatase (Dako) was incubated for 30 minutes.
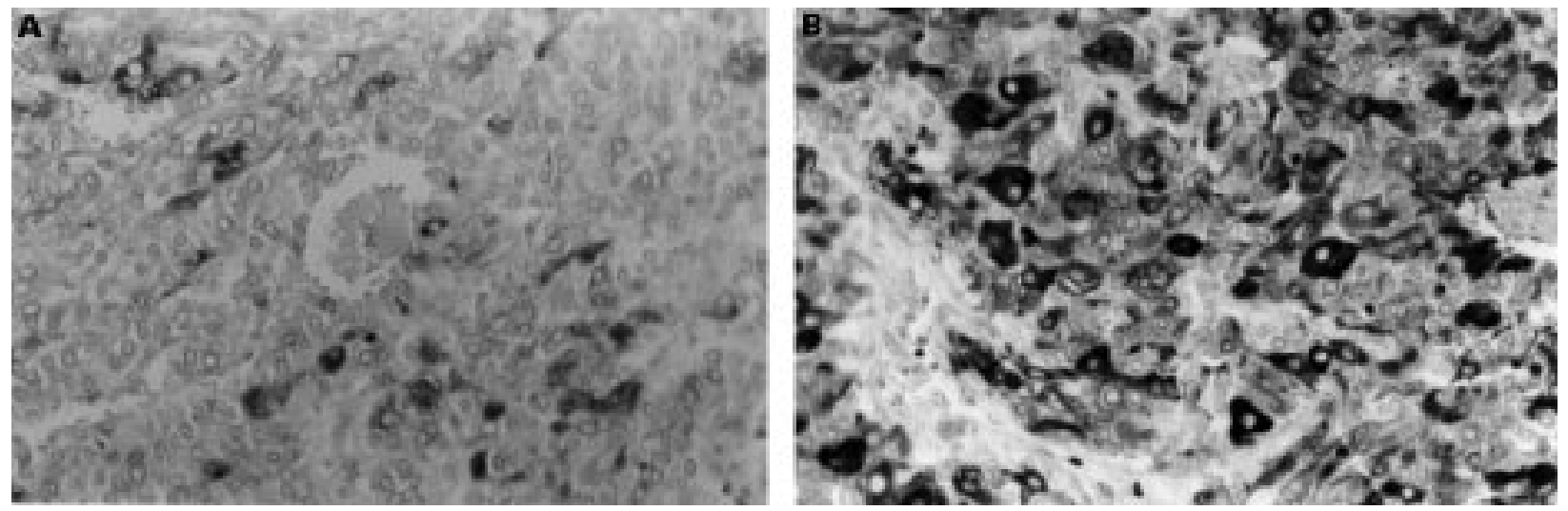

Figure 1 Chromogranin immunostaining of a case of phaeochromocytoma. (A) Paraffin section cut in 1992. (B) Same area of the same block recut in 1996. A strong decrease of staining is noted on years old slides. 

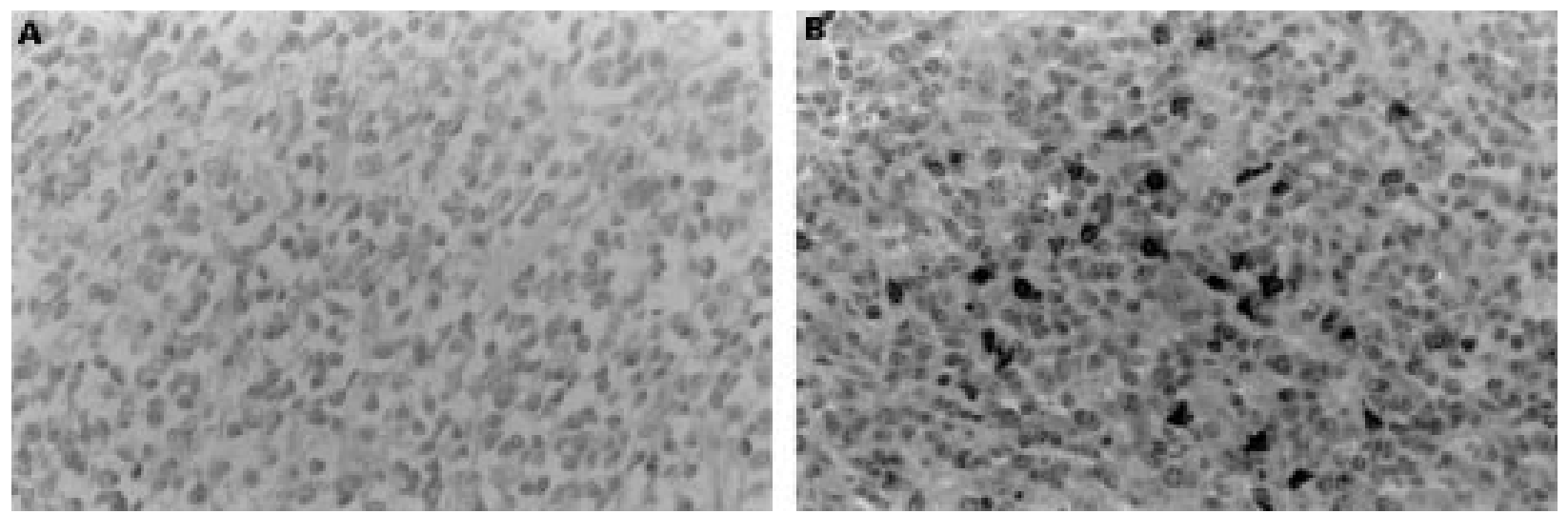

Figure 2 CD3 immunostaining of a case of B cell lymphoma. (A) Paraffin section cut in 1987. (B) Same area of the same block recut in 1996. Reactive $T$ cells are not stained on older slides (counterstained with haematoxylin).

-Peroxidase substrate was incubated for 10 minutes and alkaline phosphatase substrate was incubated for 30 minutes.

After immunostaining, slides were counterstained with haematoxylin and coverslipped.

\section{REPRODUCIBILITY}

For antibodies with differences of staining, interexperiment reproducibility was tested whenever a sufficient number of old slides was available (this was done for anti-chromogranin, anti-vimentin, anti-CD3, and anti-OR antibodies). Intraexperiment reproducibility was tested by processing simultaneously three recent slides for anti-chromogranin, antivimentin, and anti-CD3.

Reproducibility was not tested for the antibodies showing identical staining in almost all cases.

\section{IMMUNOSTAINING ANALYSIS}

Comparisons between old and recent slides were performed by one observer (PB) who was blinded with regard to the length of storage.

We analysed only cases for which at least one of the two slides showed significant stainingthat is, very weakly stained cases were rejected.

The following staining characteristics were analysed: intensity, distribution, number of stained cells, and background. No difference was noted in any case in terms of distribution of staining or number of stained cells, except when the staining was completely abolished.
We therefore kept only the intensity of staining and the presence/absence of background for the final comparisons.

Staining intensity was scored as:

-no staining (as compared to the other slide) -strongly reduced (as compared to the other slide)

-moderately reduced (as compared to the other slide)

-no significant difference (as compared to the other slide).

\section{Results}

Results for years old slides, one year old slides, and three months old slides are shown in tables 2 and 3.

ANTI-CHROMOGRANIN STAINING

Staining intensity with anti-chromogranin antibody was weaker on most years old slides as compared to recent slides (fig 1). The difference was moderate in one case and strong in three cases. One year old slides also showed a slightly reduced staining in three of six tested cases. For three months old slides, there was almost no difference.

ANTI-CD3 STAINING

In five of nine cases, staining intensity with anti-CD3 antibody was weaker on years old slides compared with recent slides. The staining intensity was strongly decreased in one case and absent in another (fig 2). An increase of
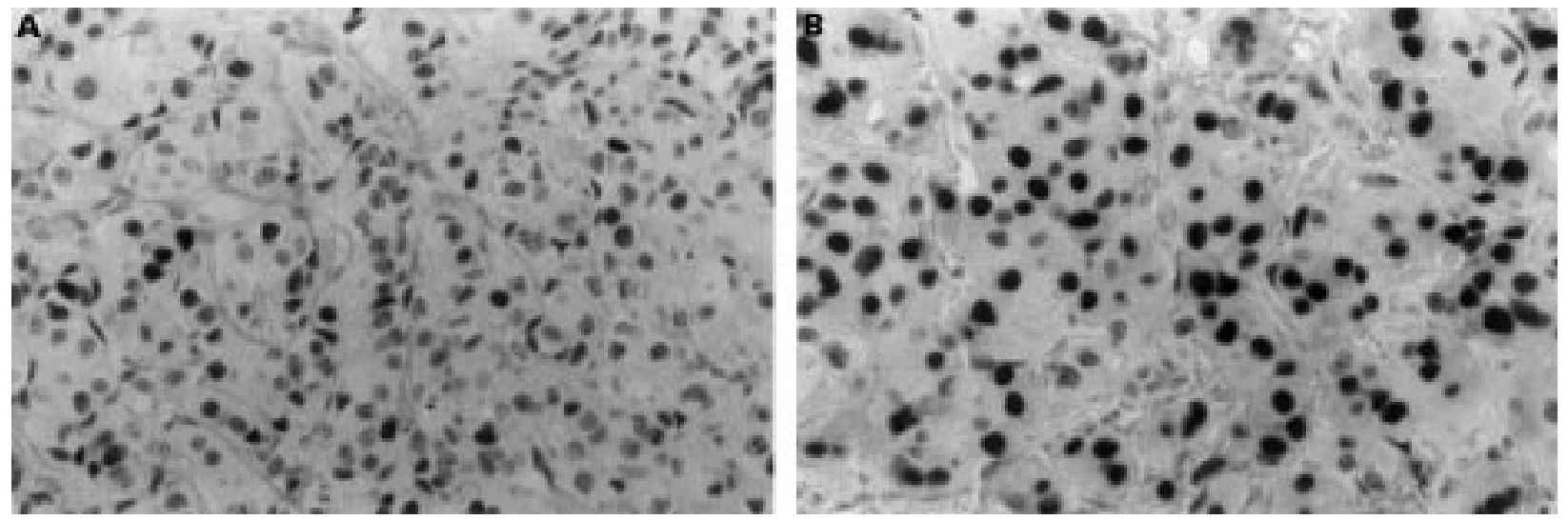

Figure 3 Oestrogen receptor (OR) immunostaining of a case of breast carcinoma. (A) Paraffin section cut in November 1996. (B) Same area of the same block recut in February 1997. Nuclear staining of tumour cells is weaker on older slides. 

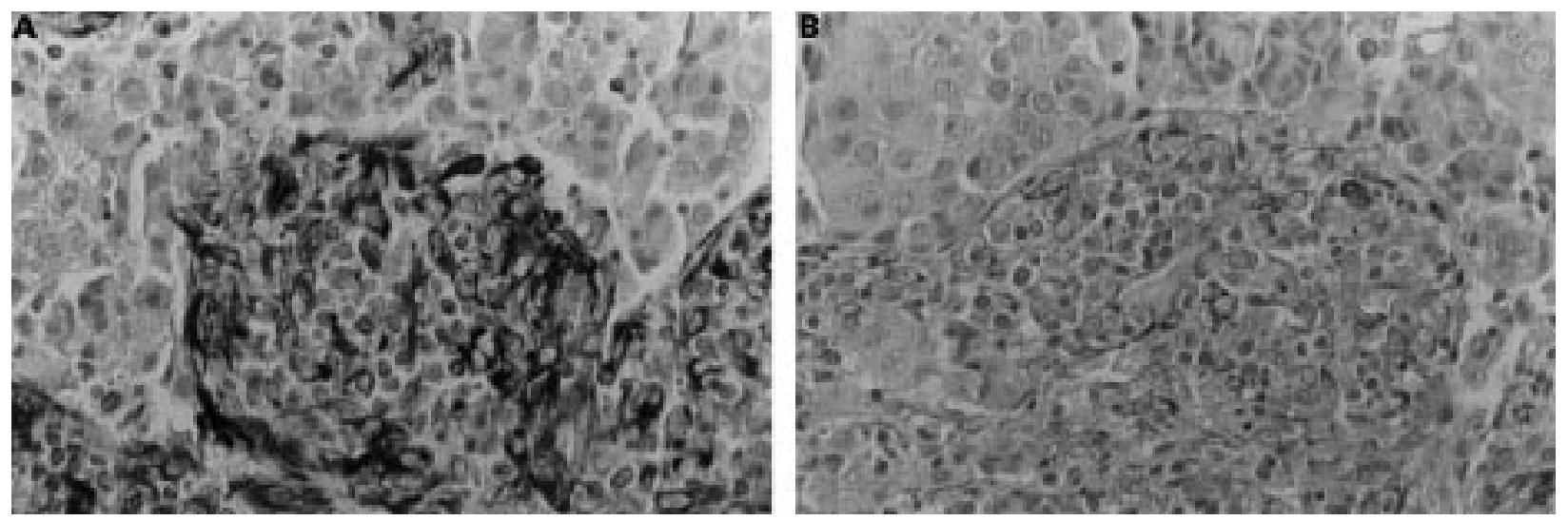

Figure 4 Vimentin immunostaining of a case of breast carcinoma. (A) Paraffin section cut in 1991. (B) Same area of the same block recut in 1996. Stromal cells show a significantly stronger staining in older slides.

background was noted in five cases. Only slight differences were noted on one year old slides and there was no significant difference with three months old slides.

ANTI-OR STAINING

Among six cases using one year old gelatin coated slides, we showed a lack of immunostaining in four cases and a strong decrease in another compared with recent slides. A loss of staining or a weaker staining (fig 3) was also noted in five of eight cases in three month old slides compared with recent slides.

ANTI-VIMENTIN STAINING

Immunostaining intensity for vimentin was significantly stronger in years old slides than in recent slides (fig 4). This was noted in six of eight cases.

OTHER ANTIBODIES (KL1, EMA, SMA, PS100, CD45, CD20, CD30)

No consistent difference of immunostaining was noted between years old slides and recent slides.

\section{Discussion}

We have compared the immunoreactivity of paraffin slides stored for three months to 10 years with paraffin slides prepared less than seven days before immunostaining. We found significant differences for anti-chromogranin, anti-OR, anti-CD3, and anti-vimentin antibodies.

When we initiated this study, no previous published report was available and we started our work with years old slides (three to 10 years) with the idea that if we did not find differences with such old slides, there was no need to investigate shorter periods of storage. Our preliminary results showed that something indeed happened during the storage period and we decided to extend our work to three months and one year storage duration.

We never stored unstained slides on a systematic basis in our department. Therefore we were able to find in our files only a few cases for which unstained slides had not been used at the time of diagnosis.

One recent report ${ }^{3}$ has studied the immunoreactivity of unstained slides for p53, OR, factor VIII, and bcl 2 in breast cancer. The authors found in most cases a strong decrease of p53 immunoreactivity after 12 weeks. A significant decrease of OR, factor VIII, and bcl2 immunoreactivity was also noted. Those findings have raised questions about the range of antigens and tissues for which immunostaining changes could be a potential problem.

We studied a panel of 11 antibodies that are commonly used in pathology laboratories. In the pathological research area, those antibodies are most often used before doing further immunohistochemical analyses. We have shown that three antigens that are located respectively in the nucleus (OR), the cytoplasm (chromogranin), or within the cell membrane (CD3) may be affected by this problem. Since the storage induced problems are not limited to antigens with a specific subcellular localisation, it is likely that many other antigens could be also altered, even if seven of 11 antibodies we tested did not show significant changes.

One surprising finding was the moderate increase of immunostaining for vimentin on years old slides. This favours the hypothesis that long term storage does not necessarily induce antigen loss but rather antigen alteration.

Regarding the range of tissues that may be affected by storage, we showed that not only breast tissue but also neuroendocrine tumours and lymphomas could show antigen alteration.

We have no explanation for the fact that some antigens are altered by storage and some are not. Antigen retrieval techniques with microwave heating ${ }^{4}$ were not very efficient in Jacob's work. ${ }^{3}$ Neither could we restore OR immunostaining with microwave heating in several cases. However, Kato et al reported a beneficial effect of microwave pretreatment of months old unstained slides for p53 immunostaining of colon cancers. ${ }^{5}$

Antigen degradation occurs in unstained slides - that is, in $5 \mu \mathrm{m}$ sections-while degradation does not seem to occur (or at a very low level) in paraffin blocks. It has been suggested that exposure of the section to air might be related to antigen alteration. However, as Jacobs et al reported, ${ }^{3}$ additional paraffin coating of the slides did not significantly diminish antigen loss. Storage temperature may be more important, as $4^{\circ} \mathrm{C}$ storage seemed to prevent 
immunostaining decrease to some degree in Jacob's report. ${ }^{3}$ The type of tissue fixation may be very important as well, but its participation in antigen loss/preservation in unstained slides has not yet been investigated.

Antigen alteration on stored slides is not a problem for daily practice in pathology, since immunostaining for patient diagnosis is usually performed on one or two day old slides. Antigen alteration could be a real problem for retrospective work, however. This is especially the case for studies that involve patients from several institutions. Paraffin blocks are often referred to a single pathology department. That department is usually asked by the referring institution to send the block back as soon as possible, as this may have medicolegal implications. ${ }^{6}$ It is therefore common practice in pathology departments involved in research protocols to store unstained paraffin slides for future use. Unstained slides are rarely stored for years before immunostaining, but it is clear that only a few weeks of storage may induce antigen alteration, particularly for p53 and OR.

Use of unstained slides that have been stored for various durations may explain in part some heterogeneous immunohistochemical results within studies or between different studies.?

When the use of stored unstained slides cannot be avoided, we suggest that the first step for the investigators should be to demonstrate that the antigen-antibody reaction they want to study is not altered on their oldest slides compared with recently cut sections. That first methodological step should be done for each study using stored unstained slides, as antigen loss is likely to depend on the type of tissue, the type of fixation, and the immunohistochemical procedure used, these being extremely variable among institutions.

Antigen alteration in stored unstained paraffin slides will certainly have to be taken into account in the future to increase reliability and quality of immunohistochemical studies.

We wish to thank Mr Rocher for technical assistance in preparing the photographs.

1 Bratthauer GL. Immunocytochemical methods and protocols. Preparation of frozen sections for analysis. In: Javois LC, ed. Methods in molecular biology, vol 34. Totowa, New Jersey: Humana Press, 1994.

2 Prioleau JE, Schnitt SJ. p53 Antigen loss in stored paraffin slides [letter]. N Engl f Med 1995;332:1521-2.

3 Jacobs TW, Prioleau JE, Stillman IE, et al. Loss of tumour Jacobs TW, Prioleau JE, Stillman IE, et al. Loss of tumour
marker-immunostaining intensity on stored paraffin slides marker-immunostaining intensity on stored paraffin

$4 \mathrm{Shi}$ SR, Key ME, Kalra KL. Antigen retrieval in formalin-fixed, paraffin embedded tissues: an enhancement method for immunohistochemical staining based on microwave oven heating of tissue sections. F Histochem Cytochem 1991;39:741-8

5 Kato J, Sakamaki S, Niitsu Y. More on p53 antigen loss in stored paraffin slides [letter]. $N$ Engl f Med 1995;333: 1507-8.

6 Mills SE, Fechner RE, Frierson HF, et al. Guardians of the wax .... and the patient [editorial]. Am $\mathcal{F}$ Clin Pathol wax $\ldots$ and the

7 Henson DE. Loss of p53 immunostaining intensity in breast cancer [editorial]. F Natl Cancer Inst 1996;88:1015-16. 